\title{
A Generalized Model for Indoor Location Estimation Using Environmental Sound from Human Activity Recognition
}

\author{
Carlos E. Galván-Tejada ${ }^{1, *}$, F. E. López-Monteagudo ${ }^{1}$, O. Alonso-González ${ }^{2}$, \\ Jorge I. Galván-Tejada ${ }^{1}$, José M. Celaya-Padilla ${ }^{3}$ (D), Hamurabi Gamboa-Rosales ${ }^{1}$ (iD), \\ Rafael Magallanes-Quintanar ${ }^{1}$ and Laura A. Zanella-Calzada ${ }^{1}$ \\ 1 Unidad Académica de Ingeniería Eléctrica, Universidad Autónoma de Zacatecas, Jardín Juarez 147, Centro, \\ 98000 Zacatecas, Zac, Mexico; eneldolm@uaz.edu.mx (F.E.L.-M.); gatejo@uaz.edu.mx (J.I.G.-T.); \\ hamurabigr@uaz.edu.mx (H.G.-R.); tiquis@uaz.edu.mx (R.M.-Q.); lzanellac@uaz.edu.mx (L.A.Z.-C.) \\ 2 Unidad Academica de Ingeniería I, Universidad Autónoma de Zacatecas, Jardín Juarez 147, Centro, \\ 98000 Zacatecas, Zac, Mexico; omero.alonso@uaz.edu.mx \\ 3 CONACYT, Universidad Autónoma de Zacatecas, Jardín Juarez 147, Centro, 98000 Zacatecas, Zac, Mexico; \\ jose.celaya@uaz.edu.mx \\ * Correspondence: ericgalvan@uaz.edu.mx
}

Received: 2 December 2017; Accepted: 1 February 2018; Published: 27 February 2018

\begin{abstract}
The indoor location of individuals is a key contextual variable for commercial and assisted location-based services and applications. Commercial centers and medical buildings (e.g., hospitals) require location information of their users/patients to offer the services that are needed at the correct moment. Several approaches have been proposed to tackle this problem. In this paper, we present the development of an indoor location system which relies on the human activity recognition approach, using sound as an information source to infer the indoor location based on the contextual information of the activity that is realized at the moment. In this work, we analyze the sound information to estimate the location using the contextual information of the activity. A feature extraction approach to the sound signal is performed to feed a random forest algorithm in order to generate a model to estimate the location of the user. We evaluate the quality of the resulting model in terms of sensitivity and specificity for each location, and we also perform out-of-bag error estimation. Our experiments were carried out in five representative residential homes. Each home had four individual indoor rooms. Eleven activities (brewing coffee, cooking, eggs, taking a shower, etc.) were performed to provide the contextual information. Experimental results show that developing an indoor location system (ILS) that uses contextual information from human activities (identified with data provided from the environmental sound) can achieve an estimation that is $95 \%$ correct.
\end{abstract}

Keywords: indoor location; human activity recognition; context information; CAD; random forest; machine learning algorithms

\section{Introduction}

The physical indoor location of a user has become an important context variable because it is fundamental information that is needed to increase the capabilities of other systems to offer location-based services (LBSs) and improve the user's situation [1]. Therefore, the research topic of indoor location systems (ILSs) has been constantly improved, especially with the availability of mobile devices such as smartphones, which include sensors that can be used to develop indoor location estimator (ILE) systems with different technologies [2].

Several approaches have been proposed to develop ILEs, depending on the availability of technological devices and/or indoor location conditions. For instance, the use of radio waves that 
include technologies such as Bluetooth, radio frequency identification (RFID), ultrasonic sensors, and Zigbee, among others [3-5], which uses available radio signals generated by other devices in the environment [6]. These approaches have also been combined to develop robust ILS, taking advantage of devices that include more than one sensor (e.g., smartphones, as mentioned in $[7,8]$ ). The combination of these technologies has also been considered $[7,8]$. These technologies have allowed the development of well-accepted proposals based on these technologies, such as Active Badge [9], Active bat [10], Cricket [11], LANDMARC [12], Bluepos [13], LOSNUS [14], and CLIPS [15]. However, these approaches require a dedicated infrastructure, and in most of them the position of the devices is used to calculate the final indoor location. Thus, they are impractical for deployment in the real world. Additionally, the lack of scalability is another disadvantage, because the dedicated infrastructure requires devices to be added in order to increase the coverage of these systems.

To solve this issue, other approaches make use of signals that are naturally in the environment [6]. These natural signals (e.g., sound, light, geomagnetic, etc.) can be used without deploying dedicated infrastructure or ILSs. One example is geomagnetic signal, which can be detected by smartphones using the built-in magnetometer sensor [16,17]. Haverinen et al. [18] propose that the position should be identified using the Monte Carlo localization approach. For this dynamic localization in an indoor environment (corridors in a building), magnetic field observations from an unknown position, the comparison between the magnetic field variations, and the known patterns are used. Gozick et al. [19] propose another approach using the variations in the geomagnetic field caused by structural steel building elements. These variations can be modeled to estimate the user location. Other approaches use light as an information source to estimate the location of the user. Randall et al. [20] proposed the use of solar cells, and recorded the patterns as a fingerprint to recognize the location of the user. The deployment of this proposal needs no infrastructure changes; however, location estimation is calculated as a trajectory and not as a punctual location, and standard solar cells are used to register luminosity (intensity of light) as well as their typical use to collect energy. Finally, environmental sound is proposed as another information source in several works. One well-known example of this is presented by Vildjiounaite [21], who proposed the use of $10 \mathrm{~s}$ of the environmental sound and using this as a "fingerprint". Then, they calculate the spectrum for each fingerprint and use some spectral frequencies features. Delgado-Contreras et al. [22] presented an approach for location classification that does not need to have an explicit information about locations to be identified. Instead, they propose the use of 62 audio features grouped in temporal, frequency, and statistical features to describe the signal. They obtained $91.42 \%$ accuracy using a support vector machine (SVM) algorithm to estimate the location.

Other approaches propose the merge of signals with contextual information; for instance, Chun $\mathrm{Zu}$ et al. [23] proposed an approach to indoor human daily activity recognition which combines motion data and location information, where location is a context information and an accelerometer provides raw data from the user movements using Bayes' theorem to fuse the context and accelerometer data. In their work, Avgoustinos Filippoupolitis et al. [24] designed and evaluated an activity recognition system composed of a smart watch, enhanced by contextual location information acquired from Bluetooth Low Energy (BLE) beacons. They claimed a classification accuracy ranging from $92 \%$ to $100 \%$. An interesting work aimed at indoor location using contextual information was put forth by Sheng Guo et al. [25]; they proposed location estimation combining pedestrian dead reckoning, human activity recognition (HAR), and landmarks to acquire accurate indoor localization information. Xiaomu Luo et al. [26] proposed an indoor tracking and activity recognition using a wireless sensor network (WSN), where the sensor nodes within the WSN consist of pyroelectric infrared (PIR) sensor arrays. The approach uses a two-layer random forest (RF) classifier to identify the user movements and activity. They claimed an error of about 0.85 meters and a 92\% HAR. Nevertheless, these approaches keep the main constraint that depends on dedicated infrastructure (for instance BLE beacons) and/or specific proposed devices to track the user movements (accelerometers, pyroelectric sensors, and smart watch among others), which implies that they cannot be deployed in many environments. 
There are approaches that instead of measuring distance in terms of coordinates in a plane $x, y$, they propose the use of labels to identify certain rooms; for instance, Ghourchian et al. [27] proposed an approach that utilizes WiFi-enabled devices inside of different rooms to describe it on the basis of these WiFi devices such as laptops, smartphones, smart TV, and others. In their scenario, they labeled the rooms with a consecutive number, i.e., "Room 1", "Room 2", and so on; however, several devices can be moved easily to modify the WiFi description of a room. Crespo et al. [28] proposed a semantic navigation in indoor environments, describing rooms as "places" that have particular objects or tasks that will help to identify each room; this proposal uses two main elements: an ontology proposed and environment information included in a relational database. Room semantics identification using acoustic monitoring Mel-frequency cepstral coefficients (MFCCs) to model the environmental sound is presented by Ahmed Shah et al. [29], who propose the use of support vector machines (SVMs) to classify the MFCCs that describe room semantics; nevertheless, a Fourier depending feature such as MFCC combined with an SVM algorithm can lead to a high computational cost. These approaches identify rooms to offer indoor location systems; however, current user activity is not relevant to these proposals. Therefore, in this paper, we propose a context information ILS which relies on the human activity recognition (HAR) process and how it can describe the location with environmental sound as information source, based on contextual information to estimate the user's location in an indoor environment. In this approach, the main goal is to find the user's location in terms of descriptive location "labels" (e.g., "kitchen"), and not a particular point in a coordinate system. However, this location description is enough to provide location based services (LBSs). Therefore, instead of measuring the error in distance from the current location and the estimated one, this is measured as in a classification problem-estimated room against the actual room (true positives and true negatives). This allows the performance of the ILS to be expressed in several numerical metrics, such as sensitivity, specificity, and recall. A confusion matrix can be calculated and other visualization figures can be developed, such as a receiver operating characteristic (ROC) curve.

There are two important goals in our study: to provide evidence that human activity as context information can describe the location of the user in an indoor environment, and secondly, to find statistical features that explain the behavior of the signals to develop an indoor location system based on context information about the activity that is performed, with low computational cost that allows us to implement our system in portable devices such as smartphones without the need of adding infrastructure to the environment. This ILS must be a general model (i.e., a model that does not depend on a particular user [30,31], device, or specific characteristics of the signal that feeds the model), with the aim of avoiding a costly training phase in order to be an easily-implemented development.

In order to perform the context information extraction, using HAR with sound as information source, statistical features presented by Galván-Tejada et al. [32] were used. In our proposal, we construct a context sound fingerprint with $10 \mathrm{~s}$ of HAR that is carried out in an indoor environment. With this context, the sound fingerprint can be inferred in the indoor room where a certain activity is performed. This approach allows us to be independent of the constraints occurring in other approaches using other natural signals as mentioned before.

The main contribution of this work is the use of contextual information extracted from human activity recognition to estimate the user's location in an indoor environment, without the need of adding devices to the user or to the environment. This approach uses sound data to estimate the location, which is present in any indoor environment and thus there is no need to generate or modify infrastructure.

This paper is organized as follows: after this introduction, in Section 2 the environmental sound data set is described in detail, as well as methods used to develop the ILS. In Section 3, the experiment using sound data from human activities and results from the experiment are shown. In Section 4, discussion and conclusions about this work are presented, and finally in Section 5 the future work is briefly described. 


\section{Materials and Methods}

In this section, the environmental sounds that comprise the data set of human activities and their locations is described in detail, and then the methods applied to develop the indoor location estimation (ILE) model are presented next.

\subsection{Data Set Description}

The human activity sounds data set is comprised of ten activities and several sounds from environmental noises tagged as non-activity. These activities are commonly performed in a residential home. Brewing coffee, cooking meat, boiling eggs, dish washing, and using the microwave oven are performed at kitchen; taking a shower, hand washing, and teeth brushing occur in the bathroom; chewing food in dining room; and finally, reading a book and sounds not related to the mentioned activities, tagged as no activity in the room for resting. A brief description of the activities and the residential rooms are shown in Table 1. It is worth mentioning that kitchen and bathroom have four activities, where running water has similar background sound, adding complexity to the ILE problem. All environmental audio recordings tagged by activity and location are available on the AmiDaMi research group page (http://ingsoftware.reduaz.mx/amidami).

Table 1. Brief description of activities used to describe each of the studied rooms.

\begin{tabular}{|c|c|c|}
\hline Location & Activity & Description of Action Recorded \\
\hline \multirow{5}{*}{ Kitchen } & Brewing coffee & $\begin{array}{l}\text { Brewing coffee from putting a coffee pot on the stove to turning } \\
\text { off the stove or coffee machine turning from on to off. }\end{array}$ \\
\hline & Frying meat & From putting meat into the frying pan to turning the stove off. \\
\hline & Cooking eggs & From cracking the egg to finishing with it cooked. \\
\hline & Using microwave oven & From set-up time to opening the microwave oven's door. \\
\hline & Dish washing & $\begin{array}{l}\text { Dishes washed by hand individually or in groups of different } \\
\text { dishes; water noise in the background. }\end{array}$ \\
\hline \multirow{3}{*}{ Bathroom } & Taking a shower & $\begin{array}{l}\text { Taking a shower in different environments, in some cases water } \\
\text { fall was interrupted in intervals. }\end{array}$ \\
\hline & Hand washing & Washing hands with bar soap. \\
\hline & Teeth brushing & Audio clips include from opening the tap to closing it. \\
\hline \multirow{3}{*}{$\begin{array}{l}\text { Dining Room } \\
\text { Room for } \\
\text { resting }\end{array}$} & Chewing food & Sounds produced by chewing crispy potatoes and apples. \\
\hline & No activity & $\begin{array}{l}\text { No activity audio clips, which mostly comprise noises added by } \\
\text { the device used to record. }\end{array}$ \\
\hline & Reading a Book & Whispering and page changing. \\
\hline
\end{tabular}

\subsection{Recording Devices}

Sounds were recorded using several smartphones, with the aim of covering different specifications and recording qualities depending on the microphones embedded in these. Smartphone model, system-on-chip (SoC), and operating system (OS) are shown in Table 2; these features of hardware and software had an impact on the sound recording capabilities and process of each device. 
Table 2. Selected mobile phones system-on-chip and operating system.

\begin{tabular}{ccc}
\hline Smartphone & System on Chip (SoC) & Operating System \\
\hline Lanix Ilium s600 & Qualcomm Snapdragon 210 MSM8909 & Android 5.1 \\
LG G Pro Lite & MediaTek MT6577 & Android 4.1.2 \\
iPhone 4 & Apple A4 APL0398 & iOS 4 \\
iPhone 3GS & Samsung S5PC100 & iOS 3 \\
HTC One M7 & Qualcomm Snapdragon 600 APQ8064T & Android 4.1.2 \\
\hline
\end{tabular}

\subsection{Spatial Environments}

Sounds were recorded in different residential home environments in the four selected rooms: kitchen, bathroom, dining room, and room for resting, meaning different sound reflections, construction materials, background sounds, and home appliances-especially in the kitchen (in the activities of brewing coffee, cooking meat, frying eggs, and microwave oven).

\subsection{Meta-Data}

Sounds sampling rates from $8 \mathrm{kHz}$ to $44.1 \mathrm{kHz}$, with mono and stereo recordings were considered in this data set to create a representative sample of mobile phones' capabilities in the market. Additionally, it allowed the inclusion of future sounds to expand the data set with new activities in different locations. Table 3 shows the summary of meta-data for each performed activity in this dataset.

Table 3. Sounds meta-data per activity.

\begin{tabular}{cccc}
\hline Activity & Sample Rate & Encoding Format & Channels \\
\hline Brewing coffee & $8000-44,100 \mathrm{~Hz}$ & $\mathrm{~m} 4 \mathrm{a}, \mathrm{amr}$ & Stereo, Mono \\
Frying meat & $44,100 \mathrm{~Hz}$ & $\mathrm{~m} 4 \mathrm{a}$ & Stereo \\
Cooking eggs & $44,100 \mathrm{~Hz}$ & $\mathrm{~m} 4 \mathrm{a}$ & Stereo \\
Use microwave oven & $44,100 \mathrm{~Hz}$ & $\mathrm{~m} 4 \mathrm{a}$ & Stereo \\
Take a shower & $44,100 \mathrm{~Hz}$ & $\mathrm{~m} 4 \mathrm{a}, \mathrm{mp} 3$ & Stereo \\
Dish washing & $44,100 \mathrm{~Hz}$ & $\mathrm{~m} 4 \mathrm{a}$ & Stereo \\
Hand washing & $8000-44,100 \mathrm{~Hz}$ & $\mathrm{~m} 4 \mathrm{a}, \mathrm{amr}$ & Stereo, Mono \\
Brushing teeth & $44,100 \mathrm{~Hz}$ & $\mathrm{~m} 4 \mathrm{a}$ & Stereo \\
Chewing Food & $44,100 \mathrm{~Hz}$ & $\mathrm{~m} 4 \mathrm{a}$ & Stereo \\
Reading a book & $8000-44,100 \mathrm{~Hz}$ & $\mathrm{~m} 4 \mathrm{a}, \mathrm{amr}$ & Stereo, Mono \\
No activity & $8000-44,100 \mathrm{~Hz}$ & $\mathrm{~m} 4 \mathrm{a}, \mathrm{amr}$ & Stereo, Mono \\
\hline
\end{tabular}

\subsection{Data Preparation}

No processing was performed on the sound files in order to keep the variations of the original sounds in the experiment $[22,33]$. The selected activities are normally done within $10 \mathrm{~s}$ to several minutes (taking a shower); therefore, activities' sounds were trimmed into $10 \mathrm{~s}$ clips, allowing the ILS to estimate the location based on the activity every $10 \mathrm{~s}$.

\subsection{Feature Extraction}

To acquire data that can potentially summarize the sound of a given activity being performed in a certain room (i.e., kitchen, bathroom, dining room, and room for resting), a feature extraction approach was performed. Each $10 \mathrm{~s}$ clip was converted into an integer array, where each integer represented the magnitude of the sound wave at a given time. Even though all clips had the same duration, the length of the arrays that represented them varied from 80,000 to 441,000 samples, depending on the sample rate of the original recording. In the stereo recordings, features were extracted only from the left channel, and in mono recordings features were extracted from the raw signal.

From our experience [32,34], first- and second-order statistical features can summarize the behavior of different types of natural signals [6] that can be used to develop ILE systems; additionally, 
other types of problems with several approaches have been tackled with statistical features [35-37]. Therefore, the 16 statistical features listed in Table 4 were extracted from each sample of the human activities done in each selected room.

Table 4. Statistical features that were extracted from each sample.

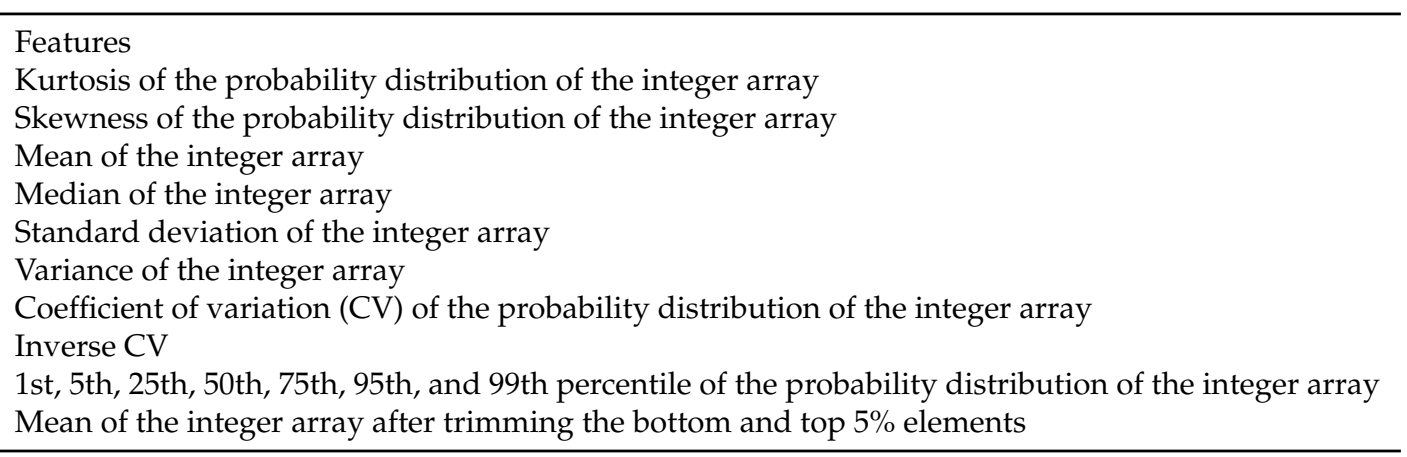

\subsection{Feature Validation}

To validate the extracted features to describe the behavior of this type of signal and be useful in the indoor location problem, a genetic algorithm approach was applied to generate a genetic rank of features to visualize the performance of each feature in the final model. This process allows the evaluation of the feature's (genes) relevance in terms of sensitivity and specificity. Based on features evaluation, an ILS model (chromosome) was developed to explore the capability of each one to explain the sound behavior, and to describe the location where the activity was done. The process of evaluating features with a genetic algorithm approach starts with a random selection of features that comprises a subset called a chromosome. Each chromosome represents a model that can predict the dependent variable with a certain level of fitness. This chromosome mutates along generations to include new genes (features) randomly and evaluate the change in terms of fitness, allowing the behavior of each feature to be evaluated. Finally, after a defined number of random evolutions of different chromosomes, a gene rank is developed which depends on the fitness achieved in the mutation process, making it possible to know the behavior and importance of each feature to model the phenomena [38].

\subsection{Model Generation with Random Forest}

In the work of Breiman et al. [39], the RF classification technique was proposed, which is a machine learning algorithm created for the solution of classification problems. This technique is based on bagging and random feature selection, and it has been commonly used in different areas (i.e., medical approaches for feature extraction and breast tumor classification) [40].

$\mathrm{RF}$ is based on a set of decision trees composed by randomly-selected features; each tree is conformed by split nodes and leaf nodes. The split nodes are the parts where these trees grow according to the evaluation of the values obtained in their random feature vector and the incoming samples, and the evaluation of the features, deciding the left or the right side of the node. The statistic of the new samples is stored in the leaf nodes in order to be used for future prediction.

The RF process has two main stages: the first stage consists of training and the second of testing. The training stage is based on the construction of multiple decision trees with the complete set of features.

Initially, these trees are constructed through the evaluation of the entire dataset; then, in the $i$ th tree (where $i$ is selected depending on the quantity of subjects), RF selects a subset of data for the training stage. This subset is randomly sampled with a replacement from the entire dataset. Using this subset of data, each node in the tree is recursively trained, starting from the root node, which is the node in the top. In the $j$ th node (where $j$ is selected depending on the quantity of subjects) is generated a function to divide the data into left and right child nodes. This process step also 
includes the improvement of randomness in the trees of the forest based on the selection of features by random sampling.

The training stage is repeated until the criteria point is reached, which is established before the algorithm starts and represents the maximum of the tree depth or a statistical value.

Finally, in the testing stage, the process is very similar to the training stage. The remaining subset of data-reserved for testing - is submitted to the forest in the root node of each tree, being classified into the left or the right child node until arriving at a leaf node, according to the learned function from the training stage. From the forest, each tree presents a prediction result based on the statistic of the training values that were initially reserved in each leaf node. The final result of the algorithm is calculated by averaging the results obtained from the prediction of each tree [41].

To validate the results, RF uses the out-of-bag (OOB) error, which is an unbiased estimate of the true prediction error that consists of testing each tree on the samples not used in the building trees stage. According to Breiman et al. [39], it was demonstrated that calculating the OOB error produces the same results in the error estimation using training and testing sets of the same size [39].

RF results will differ each time that the algorithm is performed because of the randomness set in the tree building process; nevertheless, this randomness can be specified to be always the same, obtaining specific purpose forests for certain problems [42].

This classifier is a widely used machine learning algorithm due to the simplicity in the approach of the global interpretation of the logical relation between features, values, and classes [38].

\subsection{Random Forest Model Validation}

Even when RF estimated the final model error using the OOB approach, model validation was done with a blind test, splitting the data set into two balanced partial data sets: training set and test set. From this blind test, ROCs were calculated for each room. The ROC allows the performance of a classifier to be evaluated in terms of sensitivity and specificity, revealing the ability of the model to recognize true positives (TPs, observations that actually belong to the class in which were classified) and false positives (FPs, observations that belong to another class but were classified as the current evaluated class).

A well-known metric to evaluate the performance of a classifier using a ROC is the area under the curve (AUC), which explains the general performance of the model using sensitivity and specificity data.

\section{Experiments and Results}

The activities sound data set is comprised of 64 recordings with $120110 \mathrm{~s}$ human activity sound descriptors. Table 5 details the number of recordings and $10 \mathrm{~s}$ instances per room obtained from the activities.

Table 5. Number of recordings and $10 \mathrm{~s}$ instances per room described by contextual activity recognition information.

\begin{tabular}{clccc}
\hline Location & Activity & Recordings & 10 s Instances & Total Sounds Per Room \\
\hline \multirow{5}{*}{ Kitchen } & Brewing coffee & 9 & 245 & \\
& Cooking (Meat and Eggs) & 6 & 132 & 553 \\
& Use microwave oven & 3 & 42 & \\
& Washing dishes & 6 & 134 & 590 \\
\cline { 2 - 5 } Bathroom & Take a shower & 11 & 428 & \\
& Brushing teeth & 9 & 92 & 29 \\
\multirow{5}{*}{ Dining Room } & Washing hands & 15 & 70 & 29 \\
\cline { 2 - 5 } & Chewing food & 6 & 29 & \\
\hline
\end{tabular}


The final data set had 1201 observations from all the locations, with 16 statistical features each.

To validate that all features are relevant to explain the behavior of the audio signal to describe an indoor location with the current human activity, a genetic algorithm was used to explore these features' potential. To apply the GA approach, the Galgo package was used [38]. This package implements a generic genetic algorithm approach primarily used for variable (genes) selection in random subsets (chromosomes). Galgo is a generic R software package that uses a genetic algorithms approach in order to optimize problems by a selection of features (genes) subsets (chromosomes) in partially isolated niches (environments) that warrant a non-biased genes analysis.

Settings were set to generate five genes (features) chromosomes (models); these genes represent the 16 previously-extracted features. There were 300 evolving generations, using three different algorithms as function cost: K-nearest neighbors, nearest centroid, and RF. This was done with the aim of corroborating parametric and non-parametric classification algorithms' behavior. These parameters are recommended in the literature $[38,43,44]$ to achieve a statistically significant and non-biased process. Figure 1 presents gene rank acquired after genetic algorithm process using the three proposed algorithms, in which it is shown that independently of the algorithm, time evolution quantile features are dominant features.

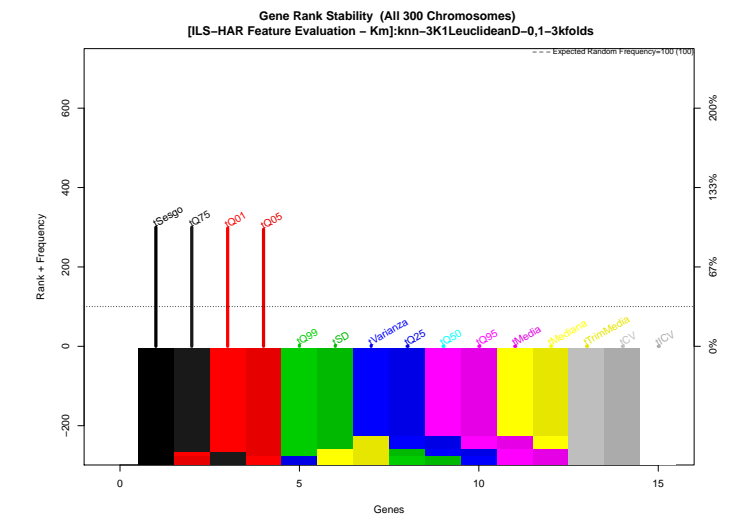

(a) Gene rank stabilization with K-nearest neighbors

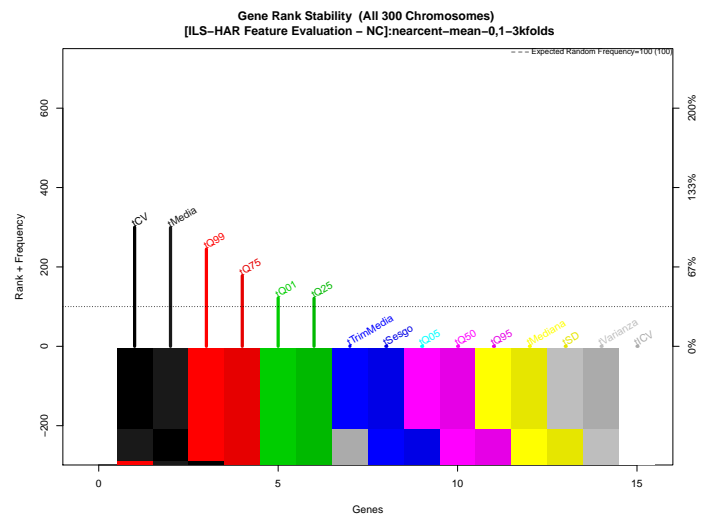

(b) Gene rank stabilization with nearest centroid

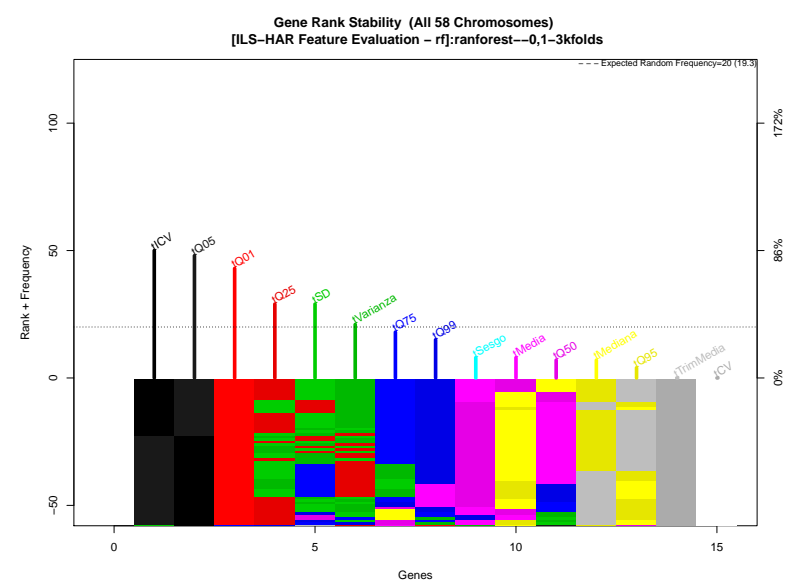

(c) Gene rank stabilization with random forest

Figure 1. Gene rank stabilization with three different algorithms as function cost.

Once the data set pre-processing and feature evaluation was done, a RF with 500 trees and classification mode was trained, using all the features as a result of the gene rank stabilization from 
the three algorithms to develop an ILS. The variation of the forest tended to zero when 500 trees were reached, as can be appreciated in Figure 2, meaning that the local optima of the forest was achieved and the location estimation had the minimum error.

\section{M@object}

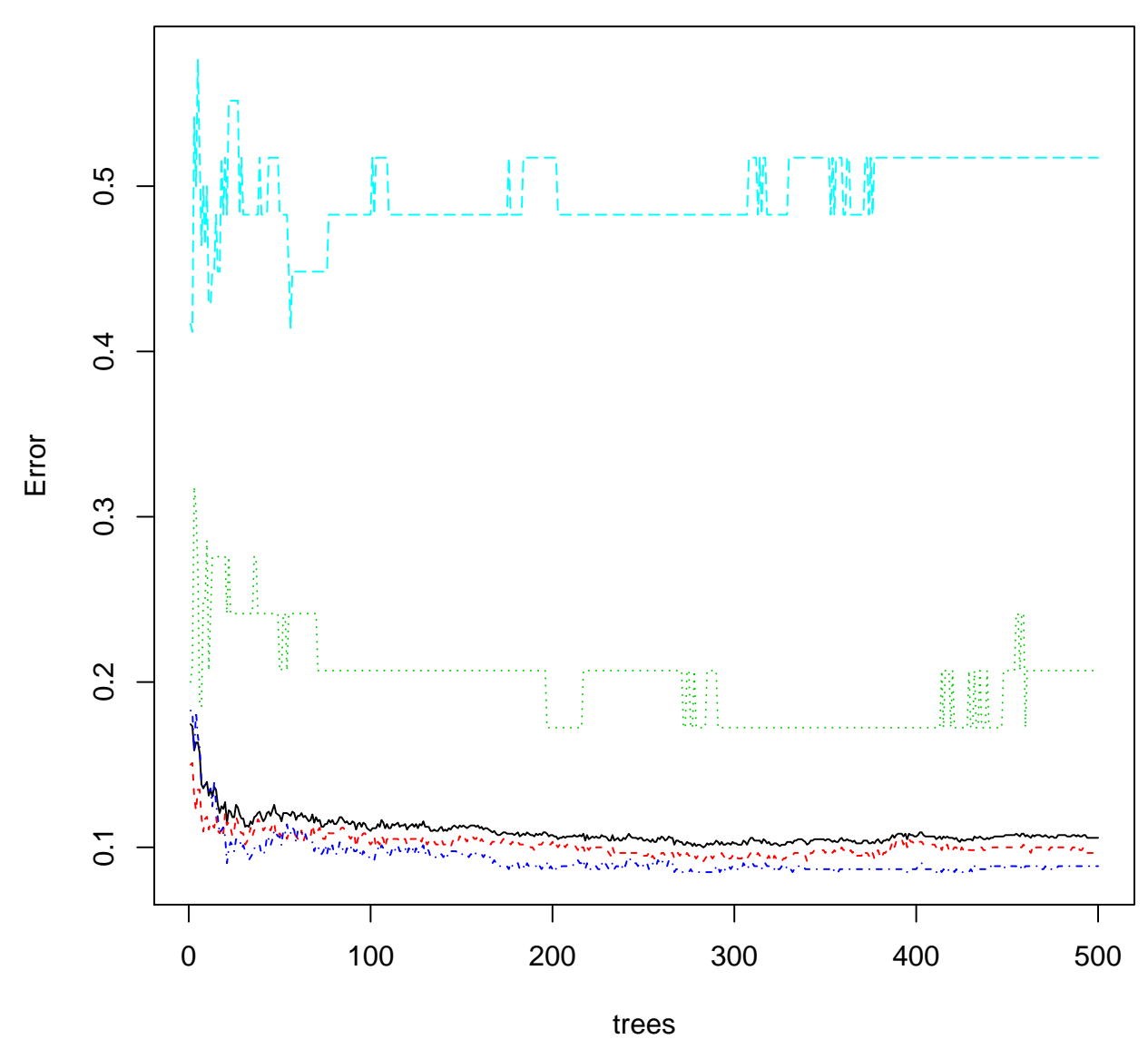

Figure 2. Random forest (RF) stabilization with 500 trees.

After this processing is done, it is possible to visualize the two principal trees and evaluate the performance and description of the model. Maximum nodes tree and minimal nodes tree are used to represent the behavior of the RF. Figure 3 shows that the final minimum error tree, after the RF creation process with the maximum number of nodes, quartiles, and percentiles features, had more weight than other statistical features, based on the potential to resume the behavior of a signal in evolutionary time form, which is consistent with the gene rank acquired by the GA approach. In addition, the minimal tree from the RF process can be seen in Figure 4; however, percentile features are still present in the root and first level branches of the tree. The meaning of this is that quantile features-which are time-independent-include relevant data to explain indoor location with the activities' sound. 


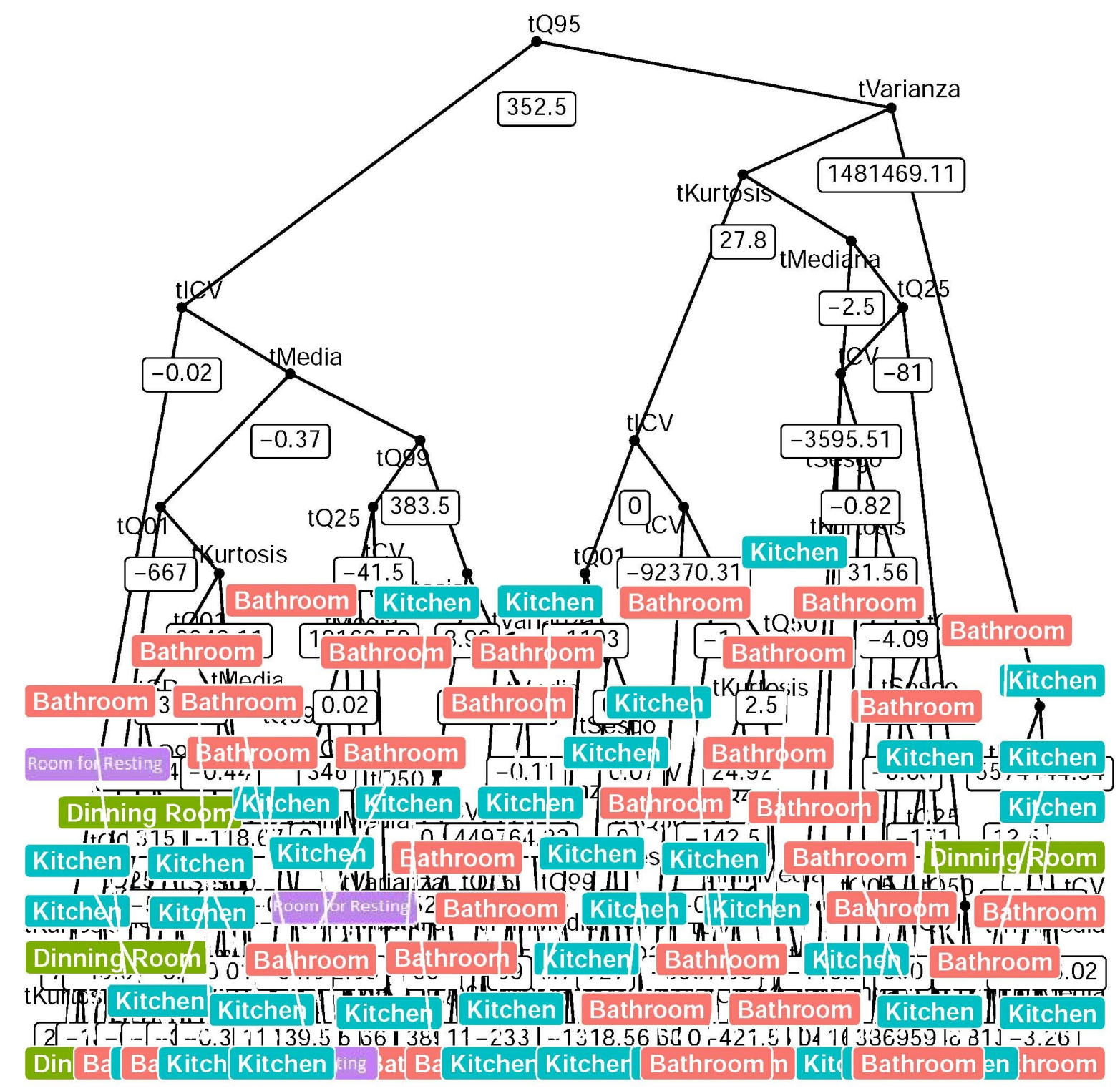

Figure 3. Minimal error tree from random forest 500 trees; all features are present. 


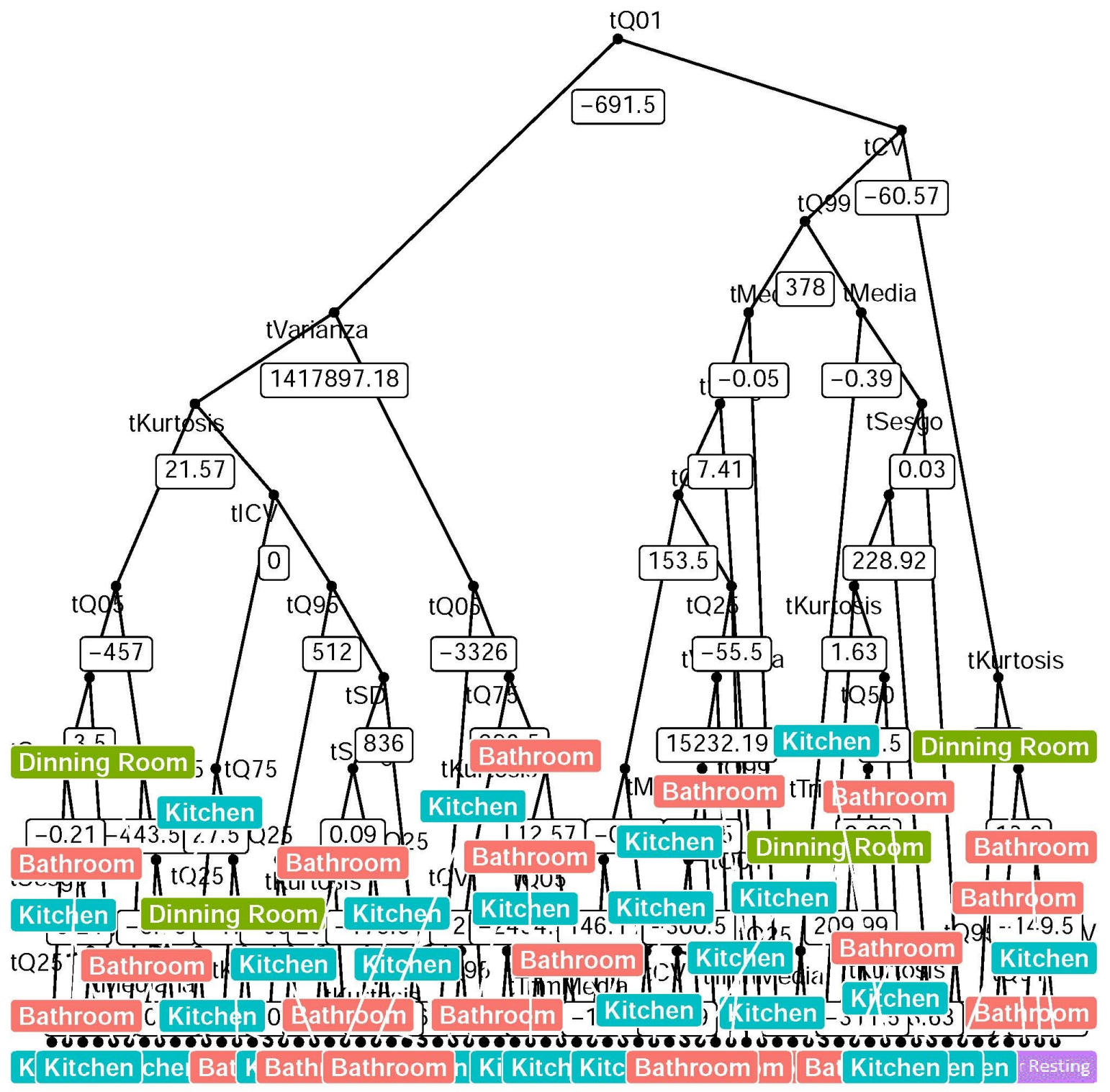

Figure 4. Minimal features tree from 500 trees generated by the random forest algorithm.

The confusion matrix acquired by the RF model is given in Table 6. The indoor location estimation model using human activity showed $8.3 \%$ percentage of error, with $92.4 \%$ sensitivity and $95.6 \%$ specificity.

Table 6. Confusion matrix of an RF with 500 trees.

\begin{tabular}{cccccc}
\hline & Bathroom & Dining Room & Kitchen & Room for Resting & Error \\
\hline Bathroom & 534 & 1 & 53 & 2 & 0.094 \\
Dining Room & 1 & 23 & 4 & 1 & 0.20 \\
Kitchen & 47 & 0 & 505 & 1 & 0.086 \\
Room for Resting & 5 & 1 & 9 & 14 & 0.51 \\
\hline
\end{tabular}

The ROC curves displayed in Figure 5 are acquired from a blind test (66\% for training and 34\% for blind test), as is recommended in literature [38]; this figure shows a true positive rate (TPR) and a 
false positive rate (FPR) for each location, with an average AUC of 0.951, which is consistent with the 0.956 from the OOB error of the RF model training.

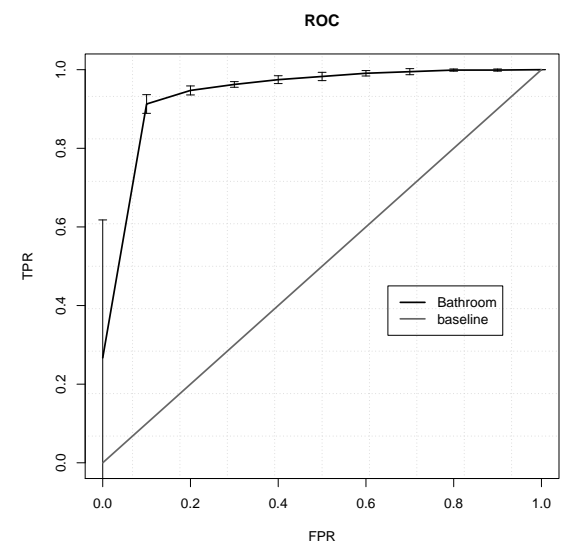

(a) Bathroom

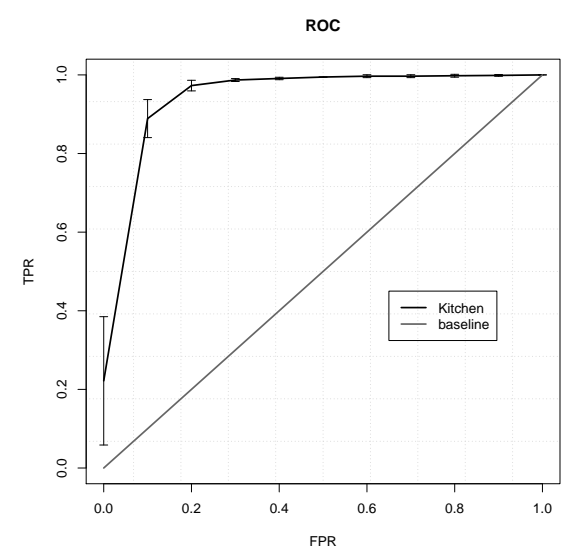

(c) Kitchen

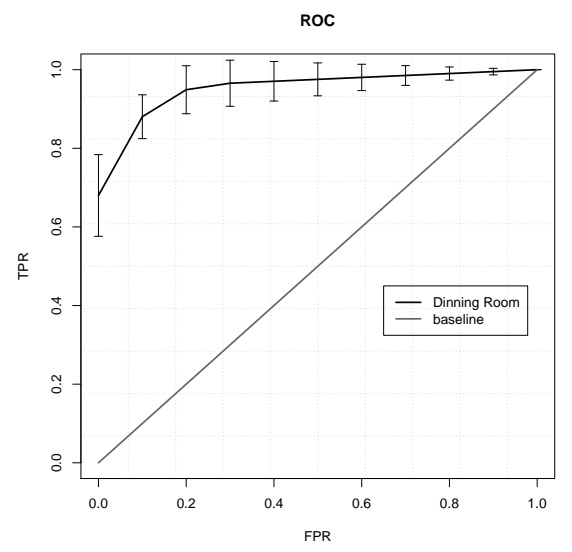

(b) Dining room

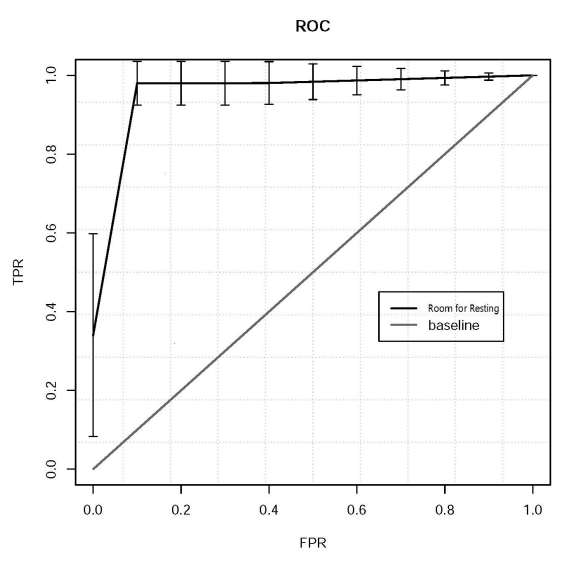

(d) Room for resting

Figure 5. Receiver operating characteristic (ROC) curves for each room.

\section{Discussion and Conclusions}

The aim of this research was to develop an indoor location estimator model using human activity recognition through activities' sound in indoor locations (i.e., kitchen, bathroom, room for resting, and dining room). The method uses feature extraction and a well-known machine learning technique (RF) that can be implemented in several platforms and deployed in different types of devices (e.g., mobile devices) to provide context information. The results presented in Section 3 allowed us to identify the following aspects to answer questions presented in Section 1:

- Human activity sound can correctly describe an indoor location: Human activity sounds have enough data that they can be used to describe indoor environments. Therefore, an indoor location estimation can be developed using human activity recognition context information with environmental sound as data source.

- Quantile statistic features correctly describe the behavior of the signal: Statistical features that are independent of time (i.e., ordered features as quantiles) can describe the behavior of the signal to estimate the location based on the human activity. Minimal and maximum trees from the RF has 
as root a quantile $n$ feature; meanwhile, descriptive statistics features tend to appear near to the final nodes (final classification).

- Context information can be used to provide LBS: Providing a system with contextual information-such as location and activity—can be useful to provide services to the user; in this case, location can be recognized with human activity that is done in a certain room in an indoor environment.

One of the most interesting points presented in this work is the use of raw data to describe contextual information (human activity) to feed an RF for indoor location estimation. This well-known classification algorithm allows us to estimate the location in $10 \mathrm{~s}$. Additionally, it can be trained with new data to describe new locations.

However, even though in this paper it is demonstrated that human activity can describe indoor locations, several activities could lead to reduce the fitness of the ILS estimation; for instance, activities that can be done in more than one room (chewing food or reading a book). This problem must be studied with a robust ILS in terms of time dependence and/or a specific activities room description based on an ontology.

Further, gene rank allows us to see that, even when all features contribute to generate the classification model, to achieve a higher AUC, a deeper analysis of features is needed, and complemented by other techniques of context or temporal inclusion.

\section{Future Work}

This study allows us to demonstrate that it is possible to develop an indoor location estimator using information from human activity; however, there are several issues that must be tackled; therefore, we propose as part of future work including other types of features (spectral evolution features), because the behavior of the quantiles and their time independence-in addition to feature selection approaches-could help to reduce the amount of features needed to develop an ILE model. Given spatial/temporal constrains, including an ontology or last-event-dependent algorithm (Petri nets, for instance) is proposed as future work. The proposed future work is:

- To study other indoor locations that can be described by human activities,

- To include spectral evolution features that are commonly used to summarize the behavior of sounds,

- To use Net Reclassification Index (NRI) as feature selection approach to promote the reduction of redundant information,

- To implement a probabilistic algorithm (e.g., Petri nets),

- To propose an ontology to add contextual information to the final estimation.

There are also other techniques to achieve indoor location, such as the use of radio signals, inertial sensors, or even using geomagnetic signal that can be fused in a multistacking proposal to increase the accuracy of the indoor location estimation. A final model will be implemented using Internet of Things-developed devices, such Arduino, raspberry, or similar to offer indoor location-based services.

Author Contributions: Carlos E. Galván-Tejada and Francisco E. López-Monteagudo, performed the study. Carlos E. Galván-Tejada,O. Alonso-González, Jorge I. Galván-Tejada performed the study design and data analysis. José M. Celaya-Padilla and Hamurabi Gamboa-Rosales contributed to materials and methods (sounds from several activities) used in this study. Carlos E. Galván-Tejada, Jorge I. Galván-Tejada, José M. Celaya-Padilla performed statistical analysis, statistical validation with critical feedback to authors. José M. Celaya-Padilla, Rafael Magallanes-Quintanar and Laura A. Zanella-Calzada contributed with the indoor environments and recorded sounds in this study. Laura A. Zanella-Calzada provide feedback from results. All authors interpreted findings from the analysis and drafted the manuscript.

Conflicts of Interest: The authors declare no conflict of interest. 


\section{References}

1. Schilit, B.; Adams, N.; Want, R. Context-Aware Computing Applications. In Proceedings of the 1994 First Workshop on Mobile Computing Systems and Applications, Santa Cruz, CA, USA, 8-9 December 1994; IEEE Computer Society: Washington, DC, USA; pp. 85-90.

2. Brena, R.F.; García-Vázquez, J.P.; Galván-Tejada, C.E.; Muñoz-Rodriguez, D.; Vargas-Rosales, C.; Fangmeyer, J. Evolution of Indoor Positioning Technologies: A Survey. J. Sens. 2017, 2017, doi:10.1155/2017/2630413.

3. Liu, H.; Darabi, H.; Banerjee, P.; Liu, J. Survey of wireless indoor positioning techniques and systems. IEEE Trans. Syst. Man Cybern. Part C Appl. Rev. 2007, 37, 1067-1080.

4. Gu, Y.; Lo, A.; Niemegeers, I. A survey of indoor positioning systems for wireless personal networks. IEEE Commun. Surv. Tutor. 2009, 11, 13-32.

5. Mautz, R. Overview of current indoor positioning systems. Geodezija Ir Kartografija 2009, 35, 18-22.

6. Galvan-Tejada, C.E.; Garcia-Vazquez, J.P.; Brena, R.F. Natural or generated signals for indoor location systems? An evaluation in terms of sensitivity and specificity. In Proceedings of the 2014 International Conference on Electronics, Communications and Computers (CONIELECOMP), Cholula, Mexico, 26-28 February 2014; pp. 166-171.

7. Galvan-Tejada, I.; Sandoval, E.I.; Brena, R. Wifi bluetooth based combined positioning algorithm. Proced. Eng. 2012, 35, 101-108.

8. Baniukevic, A.; Sabonis, D.; Jensen, C.S.; Lu, H. Improving wi-fi based indoor positioning using bluetooth add-ons. In Proceedings of the 2011 IEEE 12th International Conference on Mobile Data Management, Lulea, Sweden, 6-9 June 2011; Volume 1, pp. 246-255.

9. Want, R.; Hopper, A.; Falcao, V.; Gibbons, J. The active badge location system. ACM Trans. Inf. Syst. (TOIS) 1992, 10, 91-102.

10. Ward, A.; Jones, A.; Hopper, A. A new location technique for the active office. IEEE Personal Commun. 1997, 4, 42-47.

11. Priyantha, N.B.; Chakraborty, A.; Balakrishnan, H. The cricket location-support system. In Proceedings of the 6th Annual International Conference on Mobile Computing and Networking, Boston, MA, USA, 6-11 August 2000; pp. 32-43.

12. Ni, L.M.; Liu, Y.; Lau, Y.C.; Patil, A.P. LANDMARC: Indoor location sensing using active RFID. Wirel. Netw. 2004, 10, 701-710.

13. King, T.; Lemelson, H.; Farber, A.; Effelsberg, W. BluePos: Positioning with Bluetooth. In Proceedings of the 2009 IEEE International Symposium on Intelligent Signal Processing, Budapest, Hungary, 26-28 August 2009; pp. 55-60.

14. Schweinzer, H.; Syafrudin, M. LOSNUS: An ultrasonic system enabling high accuracy and secure TDoA locating of numerous devices. In Proceedings of the 2010 International Conference on Indoor Positioning and Indoor Navigation, Zurich, Switzerland, 15-17 September 2010; pp. 1-8.

15. Noh, Y.; Yamaguchi, H.; Lee, U.; Vij, P.; Joy, J.; Gerla, M. CLIPS: Infrastructure-free collaborative indoor positioning scheme for time-critical team operations. In Proceedings of the 2013 IEEE International Conference on Pervasive Computing and Communications (PerCom), San Diego, CA, USA, 18-22 March 2013; pp. $172-178$.

16. Kim, S.E.; Kim, Y.; Yoon, J.; Kim, E.S. Indoor positioning system using geomagnetic anomalies for smartphones. In Proceedings of the 2012 International Conference on Indoor Positioning and Indoor Navigation (IPIN), Sydney, Australia, 13-15 November 2012; pp. 1-5.

17. Han, J.; Owusu, E.; Nguyen, L.T.; Perrig, A.; Zhang, J. Accomplice: Location inference using accelerometers on smartphones. In Proceedings of the 4th International Conference on Communication Systems and Networks, Bangalore, India, 3-7 January 2012; pp. 1-9.

18. Haverinen, J.; Kemppainen, A. Global indoor self-localization based on the ambient magnetic field. Robot. Auton. Syst. 2009, 57, 1028-1035.

19. Gozick, B.; Subbu, K.P.; Dantu, R.; Maeshiro, T. Magnetic maps for indoor navigation. IEEE Trans. Instrum. Meas. 2011, 60, 3883-3891.

20. Randall, J.; Amft, O.; Bohn, J.; Burri, M. LuxTrace: Indoor positioning using building illumination. Personal Ubiquitous Comput. 2007, 11, 417-428. 
21. Vildjiounaite, E.; Malm, E.J.; Kaartinen, J.; Alahuhta, P. Location estimation indoors by means of small computing power devices, accelerometers, magnetic sensors, and map knowledge. In Proceedings of the International Conference on Pervasive Computing, Zurich, Switzerland, 26-28 August 2002; pp. 211-224.

22. Delgado-Contreras, J.R.; Garcia-Vazquez, J.P.; Brena, R.F. Classification of environmental audio signals using statistical time and frequency features. In Proceedings of the 2014 International Conference on Electronics, Communications and Computers, Cholula, Mexico, 26-28 February 2014; pp. 212-216.

23. Zhu, C.; Sheng, W. Motion-and location-based online human daily activity recognition. Pervasive Mobile Comput. 2011, 7, 256-269.

24. Filippoupolitis, A.; Oliff, W.; Takand, B.; Loukas, G. Location-Enhanced Activity Recognition in Indoor Environments Using Off the Shelf Smart Watch Technology and BLE Beacons. Sensors 2017, 17, 1230.

25. Guo, S.; Xiong, H.; Zheng, X.; Zhou, Y. Activity Recognition and Semantic Description for Indoor Mobile Localization. Sensors 2017, 17, 649.

26. Scheurer, S.; Tedesco, S.; Brown, K.N.; O’Flynn, B. Human activity recognition for emergency first responders via body-worn inertial sensors. In Proceedings of the 14th International Conference on Wearable and Implantable Body Sensor Networks, Eindhoven, The Netherlands, 9-12 May 2017; pp. 5-8.

27. Ghourchian, N.; Allegue-Martinez, M.; Precup, D. Real-Time Indoor Localization in Smart Homes Using Semi-Supervised Learning. In Proceedings of the 29th AAAI Conference on Innovative Applications, San Francisco, CA, USA, 6-9 February 2017; pp. 4670-4677.

28. Crespo, J.; Barber, R.; Mozos, O. Relational Model for Robotic Semantic Navigation in Indoor Environments. J. Intell. Robot. Syst. 2017, 86, 617-639.

29. Shah, M.A.; Raj, B.; Harras, K.A. Inferring Room Semantics Using Acoustic Monitoring. arXiv 2017, arXiv:1710.08684.

30. Garcia-Ceja, E.; Brena, R. Building Personalized Activity Recognition Models with Scarce Labeled Data Based on Class Similarities. In Ubiquitous Computing and Ambient Intelligence. Sensing, Processing, and Using Environmental Information: 9th International Conference, UCAmI 2015, Puerto Varas, Chile, December 1-4, 2015, Proceedings; García-Chamizo, J.M., Fortino, G., Ochoa, S.F., Eds.; Springer International Publishing: Cham, Switzerland, 2015; pp. 265-276.

31. Brena, R.F.; Garcia-Ceja, E. A crowdsourcing approach for personalization in human activities recognition. Intell. Data Anal. 2017, 21, 721-738.

32. Carlos, E.; Galván-Tejada, J.P.; García-Vázquez, R.F.B. Magnetic Field Feature Extraction and Selection for Indoor Location Estimation. Sensor J. 2014, 14, 11001-11005.

33. Tarzia, S.P.; Dinda, P.A.; Dick, R.P.; Memik, G. Indoor localization without infrastructure using the acoustic background spectrum. In Proceedings of the 9th International Conference on Mobile Systems, Applications, and Services, Bethesda, MD, USA, 28 June-1 July 2011; pp. 155-168.

34. Galván-Tejada, C.E.; García-Vázquez, J.P.; Galván-Tejada, J.I.; Delgado-Contreras, J.R.; Brena, R.F. Infrastructure-less indoor localization using the microphone, magnetometer and light sensor of a smartphone. Sensors 2015, 15, 20355-20372.

35. Martinez-Torteya, A.; Rodriguez-Rojas, J.; Celaya-Padilla, J.M.; Galván-Tejada, J.I.; Treviño, V.; Tamez-Peña, J. Magnetization-prepared rapid acquisition with gradient echo magnetic resonance imaging signal and texture features for the prediction of mild cognitive impairment to Alzheimer's disease progression. J. Medic. Imaging 2014, 1, 031005.

36. Celaya-Padilla, J.M.; Guzmán-Valdivia, C.H.; Galván-Tejada, C.E.; Galván-Tejada, J.I.; Gamboa-Rosales, H.; Garza-Veloz, I.; Martinez-Fierro, M.L.; Cid-Báez, M.A.; Martinez-Torteya, A.; Martinez-Ruiz, F.J. Contralateral asymmetry for breast cancer detection: A CADx approach. Biocybern. Biomed. Eng. 2017, 38, 115-125.

37. Galván-Tejada, J.I.; Celaya-Padilla, J.M.; Martínez-Torteya, A.; Rodriguez-Rojas, J.; Treviño, V.; Tamez-Peña, J.G. Wide association study of radiological features that predict future knee OA pain: Data from the OAI. In Proceedings of the Medical Imaging 2014: Computer-Aided Diagnosis, International Society for Optics and Photonics, San Diego, CA, USA, 15-20 February 2014; Volume 9035, p. 903539.

38. Trevino, V.; Falciani, F. GALGO: An R package for multivariate variable selection using genetic algorithms. Bioinformatics 2006, 22, 1154-1156.

39. Breiman, L. Random forests. Mach. Learn. 2001, 45, 5-32. 
40. Galván-Tejada, C.E.; Zanella-Calzada, L.A.; Galván-Tejada, J.I.; Celaya-Padilla, J.M.; Gamboa-Rosales, H.; Garza-Veloz, I.; Martinez-Fierro, M.L. Multivariate Feature Selection of Image Descriptors Data for Breast Cancer with Computer-Assisted Diagnosis. Diagnostics 2017, 7, 9.

41. Huang, L.; Jin, Y.; Gao, Y.; Thung, K.H.; Shen, D.; Initiative, A.D.N. Longitudinal clinical score prediction in Alzheimer's disease with soft-split sparse regression based random forest. Neurobiol. Ag. 2016, 46, 180-191.

42. Biau, G.; Devroye, L. On the layered nearest neighbour estimate, the bagged nearest neighbour estimate and the random forest method in regression and classification. J. Multivar. Anal. 2010, 101, 2499-2518.

43. Torteya, A.M.; Peña, J.G.T.; Alvarado, V.M.T. Multivariate predictors of clinically relevant cognitive decay: A wide association study using available data from ADNI. Alzheimer's Dement. 2012, 8, P285-P286.

44. Celaya-Padilla, J.M.; Rodriguez-Rojas, J.; Galván-Tejada, J.I.; Martínez-Torteya, A.; Treviño, V.; Tamez-Peña, J.G. Bilateral image subtraction features for multivariate automated classification of breast cancer risk. In Proceedings of the SPIE Medical Imaging, International Society for Optics and Photonics, San Diego, CA, USA, 15-20 February 2014; 90351T.

(C) 2018 by the authors. Licensee MDPI, Basel, Switzerland. This article is an open access article distributed under the terms and conditions of the Creative Commons Attribution (CC BY) license (http:// creativecommons.org/licenses/by/4.0/). 